\section{Response to: Propofol ADMINISTRATION BY ENDOSCOPISTS VERSUS ANESTHESIOLOGISTS IN GASTROINTESTINAL ENDOSCOPY: A SYSTEMATIC REVIEW AND META-ANALYSIS OF PATIENT SAFETY OUTCOMES}

Daza and colleagues ${ }^{1}$ performed a meta-analysis of the literature to compare the safety of the administration of propofol by nonanesthesiologists to low-risk patients (American Society of Anesthesiologists score of I or II) undergoing endoscopy procedures. The possible complications of sedation for endoscopy are well described in their article. However, their review did not differentiate between endoscopies done in hospitals and those done in out-of-hospital premises. The resources to manage complications of propofol in hospital may be significantly different than those in other facilities. Also, it is not clear from their review what standards of care were in place at the institutes where the reports included in the meta-analysis originated. The standards of care in place for the premises and the adherence to these standards by the providers could affect the data significantly.

Regulations are essential for patient safety and it is important for endoscopists to know what the requirements for sedation are and who else must be a member of the team. The Out-ofHospital Premises Inspection Program of the College of Physicians and Surgeons of Ontario was initiated in 2010 and maintains standards for the safe delivery of sedation during endoscopic procedures. ${ }^{2}$ These standards were developed through extensive consultation with physicians and surgeons who had broad experience and expertise in this work. The standards are consistently monitored and updated as the practice standards of optimum patient care evolve. Many other Canadian provinces and territories have similar standards and regulation to ensure the safety and quality of patient care.

Owing to the potential for rapid and profound changes in anesthetic depth and the lack of antagonist medications, patients who receive propofol must receive care that is consistent with deep sedation even if moderate sedation is intended. These medications must be administered by a physician qualified to provide deep sedation. The physician administering propofol does not have to be an anesthesiologist, but does need to be qualified to administer deep sedation according to regulation and have the education and experience to manage the potential medical complications.

The standards ${ }^{2}$ further specify that if the physician administering the sedation is also performing the procedure, the patient has to be attended by a second individual (physician, respiratory therapist, registered nurse or anesthesia assistant) who is not assisting in the procedure and who is trained to monitor patients undergoing sedation.

The critical issue for endoscopic procedures is not the administration of propofol by an anesthesiologist versus an endoscopist, but rather the capability of the physician administering propofol to manage its complications, the monitoring of the patient to detect complications and the resources to manage those complications.

\section{Robert Byrick, MD; Dennis Pitt, MD, MEd}

Affiliations: From the Department of Anesthesia, University of Toronto, Toronto, Ont. (Byrick); and the Department of Surgery, University of Ottawa, Ottawa, Ont. (Pitt).

Competing interests: None declared.

DOI: $10.1503 /$ cjs.011618

\section{References}

1. Daza JF, Tan CM, Fielding RJ, et al. Propofol administration by endoscopists versus anesthesiologists in gastrointestinal endoscopy: a systemic review and meta-analysis of patient safety outcomes. Can 7 Surg 2018;61:226-36.
2. College of Physicians and Surgeons of Ontario. Policies and publications [Internet]. Toronto (ON): CPSO; 2018. Available: www.cpso.on.ca/Policies-Publications (accessed 2018 Aug. 13).

\section{AUTHOR RESPONSE}

We thank Drs. Byrick and Pitt for their thoughtful letter regarding our recent publication on the topic of propofol administration by nonanesthesiologists in endoscopy. We hope to address a number of excellent discussion points they have brought forward.

In our systematic review and metaanalysis, we searched for studies that compared patient outcomes during endoscopic procedures when propofol was administered by anesthesiologists versus endoscopists. With the intent of capturing a broad population, our inclusion criteria were not restricted to procedures performed inside or outside of hospitals. Despite our initial intent, only one of five studies included patients who received endoscopies outside of a hospital facility, ${ }^{1}$ therefore our conclusions are not easily generalizable across settings. It should be noted that we identified very low airway intervention rates, which speaks to the fact that propofol is generally a safe sedative agent, particularly in patients with low comorbidities (i.e., American Society of Anesthesiologists score of I or II). The need for an airway intervention during sedation should not be primarily affected by the facility in which the endoscopy took place, but rather by the patient's comorbidities, the nature of the procedure and the person administering the sedation. For this reason, it is essential that patients who may require advanced anesthetic expertise are identified with the use of validated screening tools. We agree that it is of paramount importance that all facilities, whether inside or outside of hospitals, adhere to local standards of patient safety. 